\title{
Avaliação do conhecimento e percepção da importância de saúde bucal pelos estudantes de odontologia
}

\author{
Evaluation of knowledge and oral health value perception by dentistry students
}

\author{
RAFAeLLA BAstos LeItE ${ }^{1}$ \\ JoAna Emília AraúJo dos Santos Furtado² \\ Kátia Simone Alves dos Santos ${ }^{1}$ \\ Raquel Cristina Barboza Gomes ${ }^{1}$ \\ Carlos Augusto Galvão Barboza ${ }^{3}$ \\ Carlos Frederico de Moraes Sarmento ${ }^{1}$
}

\begin{abstract}
RESUMO
Objetivo: Avaliar o conhecimento de procedimentos de saúde bucal e a percepção da importância destes pelos estudantes dos vários períodos do curso de Odontologia da UEPB/Campina Grande, bem como, observar o impacto e as mudanças sobre as condutas clínicas do futuro profissional diante dos pacientes por eles atendidos nas clínicas da referida universidade.

Metodologia: Foi realizado um estudo transversal com 169 alunos, os quais responderam um formulário contendo perguntas relacionadas ao conhecimento, percepção da importância, práticas e mudanças de comportamento quanto à higiene bucal. A possível existência de diferenças significativas $(p<0,05)$ ou correlações ( $r$ ou $r p b \neq 0$ ) entre as variáveis estudadas foi analisada por meio dos testes estatísticos Quiquadrado, correlações de Pearson e Point bisserial.

Resultados: Todos os participantes admitiram como importante ou muito importante o conhecimento sobre saúde/higiene bucal. A maioria $(59,20 \%)$ considera satisfatória a abordagem a respeito da orientação e condutas clínicas relacionadas à motivação. Muitos dos participantes consideraram muito importante a orientação do paciente (96,34\%); apesar disso, $20,30 \%$ deles afirmaram não realizá-la durante a primeira consulta. Quanto à etiologia de certas patologias, a maior frequência de acertos está representada por alunos de períodos mais avançados, com médias entre 5,56 e 6,12. Observou-se que 77,5\% relataram mudança nos hábitos de higiene bucal, contra $22,5 \%$ que disseram não ter tido mudança.

Conclusão: Os instrumentos de higiene utilizados com maior frequência foram antisséptico, dentifrício, escova macia e fio dental.
\end{abstract}

Palavras chave: Estudantes. Saúde Pública. Higiene Bucal.

\begin{abstract}
The objectives of this study were to evaluate the knowledge of oral health procedures and the perceived importance of these students from various periods of the course of Dentistry UEPB / Campina Grande, and observe the impact and changes on the clinical management of future professional before the patients treated by them in the said university clinics. We conducted a cross-sectional study with 169 students, who answered a questionnaire containing questions related to knowledge, perceived importance, practices and behavior changes regarding oral hygiene. The possible existence of significant differences $(p<0.05)$ or correlations ( $r \neq 0$ or $r p b)$ between variables were analyzed using the Chi-square, Pearson correlations and point biserial. All participants acknowledged as important or very important knowledge about health / oral hygiene. The majority $(59.20 \%)$ considers satisfactory approach to guidance and clinical procedures related to motivation. Most participants considered very important guidance to the patient (96.34\%); nonetheless, $20.30 \%$ of them said they did not realize it during the first consultation. Regarding the etiology of certain
\end{abstract}

Professor (a) - Departamento de Odontologia da Universidade Estadual da Paraíba, Campina Grande-PB, Brasil.

Graduanda - Departamento de Odontologia da Universidade Estadual da Paraíba, Campina Grande-PB, Brasil.

Professor Doutor do Programa de Pós-graduação em Patologia oral da Universidade Federal do Rio Grande do Norte (UFRN). 
diseases, the highest frequency of hits is represented by students of the later periods, with averages between 5.56 and 6.12 . It was observed that $77.5 \%$ reported a change in oral hygiene habits, against $22.5 \%$ who said they did not have change. The hygiene instruments were frequently used antiseptic, toothpaste, soft brush and floss.

Key-words: Students. Public Health. Hygiene.

\section{INTRODUÇÃO}

Noâmbito da saúde coletiva, aeducaçãonão é apenas uma transmissão de conhecimento, devendo levar principalmente a uma mudança de comportamento e a práticas que levam à preservação e manutenção da saúde. ${ }^{1}$

Atualmente, os aspectos norteadores das ações em saúde coletiva indicam ações para além dos limites biológicos do indivíduo, consideram também o contexto social. E, dessa forma, o conhecimento vai se delineando não apenas pelo puro contato com a realidade, mas na medida do reconhecimento, compreensão e contextualização de aspectos dessa realidade, conjugados com iniciativas conducentes ao desejo de transformação. ${ }^{2}$

Em Odontologia, os procedimentos educativos apresentam uma finalidade básica, modificar o comportamento de saúde bucal dos indivíduos, pois, uma vez orientados, os pacientes tornam-se receptivos e cooperadores em relação às medidas que thes são prescritas. ${ }^{3,4}$

No Brasil, apesar do grande número de faculdades de Odontologia e da imensa quantidade de profissionais lançados no mercado a cada ano, os índices de cárie dental e doença periodontal continuam sendo motivos de preocupação. ${ }^{4,5,6}$

Já é amplamente reconhecido que a transmissão de conhecimentos sobre hábitos de higiene e alimentares do profissional para o paciente é um fator importante na prevenção de doenças bucais, como a cárie e doença periodontal. ${ }^{7,8}$ Isso levanta a possibilidade que parte do problema seja a falta de informações adequadas para os indivíduos, ou informações que são adquiridas pelos alunos de Odontologia durante a graduação.

As informações recebidas não se limitam àquelas ancoradas em evidência científica, sendo muitas vezes ditadas também por fatores sociais, culturais, tecnológicos e econômicos/ mercadológicos. Em relação aos últimos, sabe-se que o mercado nacional apresenta várias marcas e produtos para a higiene bucal $\mathrm{e}$, muitas vezes, as tendências de consumo são motivadas pela preferência pessoal dos profissionais que atuam na área. ${ }^{9}$

São poucos os estudos que avaliam a base das orientações de higiene bucal transmitidas pelo aluno/futuro profissional para os seus pacientes, mais especificamente o conhecimento de aspectos práticos de alguns procedimentos e o grau de importância que o aluno atribui a estes.

O objetivo deste estudo é avaliar o conhecimento de procedimentos de saúde bucal e a percepção da importância destes pelos estudantes de Odontologia da UEPB/ Campina Grande. Como os participantes do estudo são alunos em diferentes estágios de formação, se tentará observar também o impacto e as mudanças sobre as condutas clínicas do futuro profissional diante dos pacientes por eles atendidos nas clínicas da referida universidade ao longo do curso.

\section{Metodologia}

Um estudo transversal foi realizado com 169 alunos de ambos os gêneros, do curso de Odontologia da Universidade Estadual da Paraíba (UEPB), na cidade de Campina Grande, Brasil. A pesquisa foi realizada no Departamento de Odontologia da UEPB. 
Para garantir a representatividade da amostra, a aplicação do questionário foi realizada na maior quantidade de alunos, que estivessem devidamente matriculados na instituição de ensino, assistindo aula regularmente e se disponibilizassem à participar do estudo. O período de coleta foi de setembro a outubro de 2012. A taxa de adesão ao estudo foi de $78,60 \%$ dos alunos, sendo a taxa de não adesão $21,40 \%$.

A coleta de dados foi realizada por um pesquisador, previamente calibrado, por meio de formulário autoaplicável criado pelo pesquisador. A parte inicial do formulário continha questões de identificação dos estudantes, caracterizando a amostra (faixa etária, sexo, estado civil, período que está cursando). As perguntas foram relacionadas ao conhecimento, percepção da importância, práticas e mudanças de comportamento dos estudantes quanto à higiene bucal (importância e forma de aquisição do conhecimento, abordagem acadêmica sobre orientação de higiene bucal, etiologia de patologias bucais relacionadas aos hábitos de higiene bucal, práticas de higiene pessoal adotadas pelos estudantes, modificações após ingresso no curso de Odontologia, importância de orientação bucal para os pacientes atendidos nas clínicas da UEPB).

Os dados foram coletados a partir das respostas dos estudantes, o formulário foi utilizado individualmente aos participantes de forma reservada, evitando qualquer tipo de constrangimento e facilitando a liberdade nas respostas.

Todos os resultados foram armazenados e avaliados usando o PASW (versão 21). Para realizar a descrição dos dados, utilizou-se a média como medida de tendência central, o desvio padrão ( $($ ) como medida de dispersão, bem como, exposição de frequências e suas respectivas porcentagens. Foram empregados ainda testes de Qui-quadrado, correlações de Pearson e Point bisserial. A escolha das correlações bisseriais deveu-se ao fato da impossibilidade de se utilizar múltiplos testes de comparação, que inflariam o erro tipo I e, por conseguinte, o erro de conjunto; e também na impossibilidade de usar, em alguns momentos, os testes Qui-quadrado, pois em algumas células das tabelas cruzadas não existiam sujeitos para a realização do teste. $\mathrm{Em}$ todas as análises inferenciais, aceitou-se significância de $p \leq 0,05$.

O estudo foi aprovado pelo Comitê de Ética em Pesquisa da Universidade Estadual da Paraíba (CAAE - 0274.0.133.000-12), em concordância com a Resolução 196/96 do Comitê Nacional de Saúde.

\section{Resultados}

Participaram desse estudo 169 estudantes; foi constatada uma perda de $21,40 \%$ da amostra (46 estudantes). Os principais motivos que contribuíram para essa perda foram a ausência do estudante e a falta de disponibilidade para responder o formulário no momento da coleta de dados. A média de idade dos alunos foi de 22,42 anos (desvio padrão de 5,01), a maioria era solteira $(91,10 \%)$ e do sexo feminino $(56,20 \%)$. Em relação à percepção a respeito da importância do conhecimento sobre Saúde/ Higiene Bucal, apenas um estudante descreveu como importante, o restante respondeu ser muito importante.

A Tabela 1 mostra a percepção do estudante quanto à abordagem ensinada sobre orientação de higiene bucal e condutas clínicas adotadas e repassadas para os pacientes em relação à motivação, durante o referido curso de graduação. A maioria considera satisfatória $(59,20 \%)$, enquanto $20,10 \%$ consideram muito satisfatória e 20,10\% regular. Apenas um estudante considerou essa abordagem como pouco satisfatória $(6,0 \%)$. 
Tabela 1. A abordagem sobre orientação de higiene bucal e condutas clínicas com os pacientes em relação à motivação.

\begin{tabular}{l|c|c|c}
\hline & Frequência & Porcentagem válida & Porcentagem acumulativa \\
\hline Muito satisfatória & 34 & 20,1 & 20,1 \\
\hline Satisfatória & 100 & 59,2 & 79,3 \\
\hline Regular & 34 & 20,1 & 99,4 \\
\hline Pouco satisfatória & 1 & 6 & 100,0 \\
\hline Total & 169 & 100,0 & \\
\hline
\end{tabular}

Quanto à relação entre a importância de orientação de higiene bucal e a realização dessa orientação para os pacientes, na primeira consulta, pelos estudantes; a maioria dos participantes considerou muito importante a orientação (96,34\%); apesar disso, 20,30\% deles afirmaram não realizá-la durante a primeira consulta do paciente. O teste de Fischer não encontrou qualquer relacionamento estatisticamente significativo $(p=0,58)$, possivelmente devido ao fato de ter sido encontrado um grande percentual para uma única resposta.

A Tabela 2 indica a relação entre forma de aquisição de conhecimento e período do curso. Os resultados evidenciaram correlações significativas de aquisição de conhecimento na graduação $(r=$ $0,33 ; p<0,01)$ e cursos, palestras, simpósios ou congressos $(r=0,26 ; p<0,01)$.

Tabela 2. Relação entre forma de aquisição de conhecimento e período do curso.

\begin{tabular}{lllll}
\hline & $\begin{array}{l}\text { Participantes } \\
(\mathrm{n})\end{array}$ & $\begin{array}{l}\text { Média de } \\
\text { Período }\end{array}$ & Desvio padrão & Efeito $(r)$ \\
\hline Graduação & 145 & 5,91 & 2,78 & $0,33^{* *}$ \\
\hline $\begin{array}{l}\text { Cursos, palestras, simpósios ou } \\
\text { congressos }\end{array}$ & 67 & 6,48 & 2,55 & $0,26^{* *}$ \\
\hline De forma autodidata & 39 & 5,21 & 3,25 & 0,06 \\
\hline Comunicação pessoal & 56 & 4,98 & 3,02 & 0,11 \\
\hline Sem conhecimento & -- & -- & -- & -- \\
\hline
\end{tabular}

** Significância estatística menor que 0,01

Ao verificar a relação entre período que os estudantes da amostra estavam cursando, e o seu conhecimento sobre a etiologia de patologias ligadas diretamente aos hábitos de higiene bucal, foi possível constatar que as patologias Cárie, Doença Periodontal e Língua Saburrosa foram as mais assinaladas pelos participantes. Estes, representados em média por alunos de períodos mais avançados, com médias entre 5,56 e 6,12. Foram encontrados resultados significativos somente para as patologias Doença Periodontal e Língua Saburrosa (Tabela 3). 
Tabela 3. Relação entre período do curso e etiologia.

\begin{tabular}{lcccc}
\hline & Participantes $(\mathrm{n})$ & $\begin{array}{c}\text { Média de } \\
\text { Período }\end{array}$ & Desvio Padrão & Efeito $(r)$ \\
\hline $\begin{array}{l}\text { Disfunção Têmporo- } \\
\text { mandibular }\end{array}$ & 5 & 3,81 & 1,79 & $-0,11$ \\
\hline Cárie & 167 & 5,56 & 2,93 & $-0,07$ \\
\hline Língua geográfica & 21 & 6,76 & 2,57 & $-0,16^{*}$ \\
\hline Tumores odontogênicos & 10 & 4,20 & 2,48 & $-0,05$ \\
\hline Doença periodontal & 154 & 5,78 & 2,85 & $-0,27^{* *}$ \\
\hline Língua saburrosa & 133 & 6,12 & 2,74 & $-0,38^{* *}$ \\
\hline Queilite actínica & 4 & 3,50 & 3,31 & $-0,10$ \\
\hline Queilite angular & 23 & 6,83 & 2,46 & $-0,17^{*}$ \\
\hline Cistos Odontogênicos & 15 & 6,33 & 2,47 & $-0,09$ \\
\hline Outro & 4 & 7 & 1,63 & $-0,08$ \\
\hline Todas as alternativas & 4 & 2,25 & 1,26 & $-0,08$ \\
\hline Nenhuma alternativa & 169 & 5,54 & 2,93 & 0,08 \\
\hline Não sei & - & - & - & - \\
\hline
\end{tabular}

* Significância estatística menor que 0,05

** Significância estatística menor que 0,01

A Figura 1 expressa a frequência da mudança de hábitos em relação às práticas de higiene bucal, após o ingresso dos estudantes no curso de Odontologia. Observou-se que $77,5 \%$ relataram mudança nos hábitos de higiene bucal, contra $22,5 \%$ que disseram não ter tido mudança.

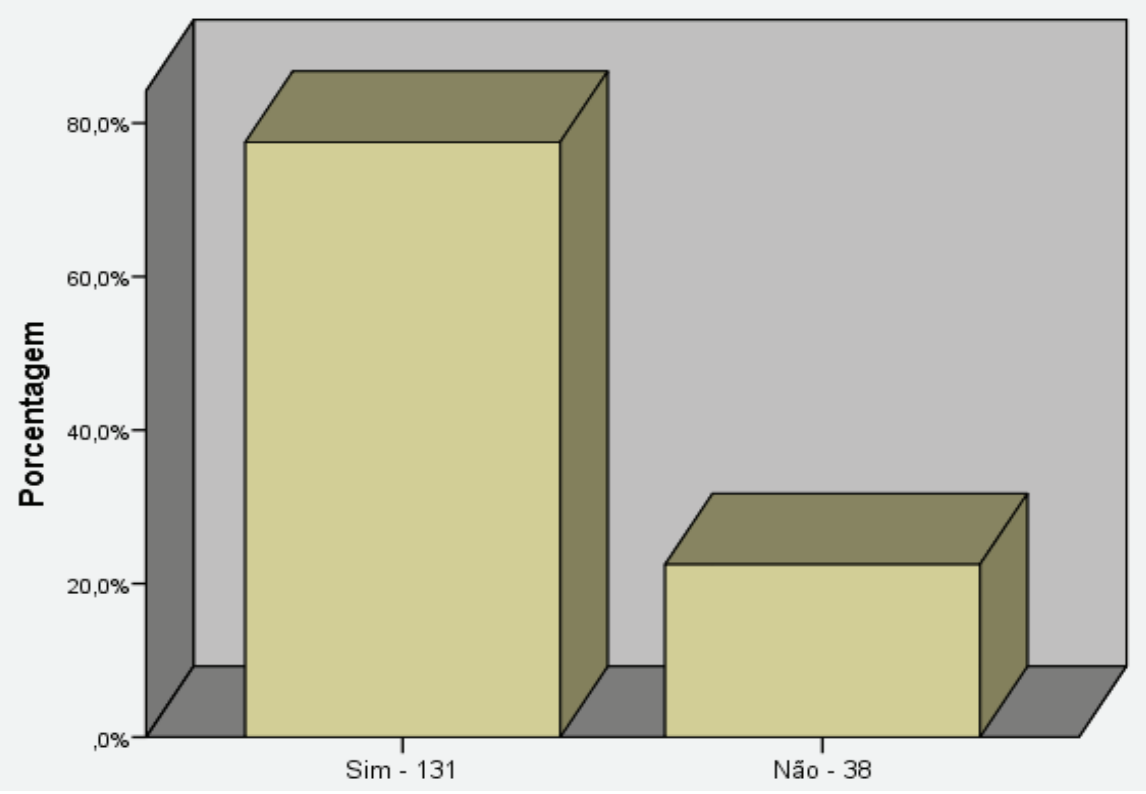

Figura 1. Ingresso no curso de odontologia muda hábitos em relação à higiene bucal. 
A Tabela 4 apresenta a relação entre o número de escovações ao dia, quantidade de vezes que o estudante utiliza o fio dental ao dia, e os instrumentos escolhidos para a realização de sua higienização. Foi encontrado relacionamento significativo apenas com o antisséptico $(r=0,16 ; p<$ $0,05)$, sugerindo que à medida que aumenta o número de escovações aumenta a chance de usar o antisséptico. Em relação ao uso de fio dental e às práticas de higiene, não foi encontrada qualquer correlação significativa. Os instrumentos utilizados pelos estudantes com maior frequência foram antisséptico, dentifrício, escova macia e fio dental, de maneira crescente.

Tabela 4. Relação entre e prática de higiene.

\begin{tabular}{|c|c|c|c|c|c|}
\hline & & $\begin{array}{c}\text { Participantes } \\
\text { (n) }\end{array}$ & Média & Desvio padrão & Efeito $(r)$ \\
\hline \multirow{6}{*}{ 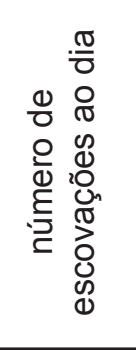 } & Escova macia & 159 & 3,32 & 0,56 & $-0,07$ \\
\hline & Escova dura & 10 & 3,13 & 0,35 & 0,07 \\
\hline & Dentifrício & 134 & 3,32 & 0,56 & $-0,04$ \\
\hline & Palito & 19 & 3,11 & 0,47 & 0,12 \\
\hline & Antisséptico & 133 & 3,35 & 0,55 & $0,16^{*}$ \\
\hline & Fio dental & 165 & 3,31 & 0,55 & $-0,09$ \\
\hline \multirow{6}{*}{ 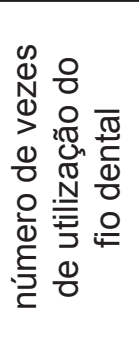 } & Escova macia & 159 & 1,72 & 0,85 & $-0,13$ \\
\hline & Escova dura & 10 & 1,20 & 0,63 & 0,15 \\
\hline & Dentifrício & 134 & 1,69 & 0,85 & 0,01 \\
\hline & Palito & 19 & 1,58 & 0,90 & 0,05 \\
\hline & Antisséptico & 133 & 1,69 & 0,84 & 0,00 \\
\hline & Fio dental & 165 & 1,69 & 0,84 & 0,01 \\
\hline
\end{tabular}

* Significância estatística menor que 0,05

\section{Dıscussão}

As transformações ocorridas no campo das políticas públicas na área da saúde têm colocado em questão, de forma cada vez mais incisiva, o perfil de formação e as práticas dos profissionais de saúde envolvidos no cuidar. A substituição do sistema dominante de atenção à saúde, centrado na doença, por modelos de atenção que valorizem a integralidade, o cuidado humanizado e a promoção da saúde deu visibilidade à necessidade de formação de profissionais com perfil capaz de atuar com qualidade e resolutividade no Sistema Único de Saúde (SUS). ${ }^{10}$ A formação de profissionais com esse novo perfil, no entanto, depende também do aluno aceitar essa nova filosofia, receber informações adequadas e acreditar que estas são importantes. O presente estudo, realizado no âmbito do curso de Odontologia da UEPB, começou a investigar essas questões.

Dessa forma, a percepção que o acadêmico de Odontologia tem a respeito da qualidade de sua formação, bem como, de itens de grande importância para essa formação humanizada é significativamente válida. A identificação da importância da Saúde Coletiva e seus variados 
aspectos, entre eles o conhecimento sobre saúde/higiene bucal e suas práticas, é praticamente universal, já que mais de $90 \%$ dos pesquisados relacionam como muito importante ou importante..$^{2,11}$

A importância do conhecimento sobre saúde/higiene bucal foi relatada como muito importante ou importante por $100 \%$ dos pesquisados, sendo que apenas um estudante a considerou importante.

Neste estudo, 79,3\% dos participantes consideraram que a abordagem, ensinada pelos docentes, sobre orientação de higiene bucal e condutas clínicas adotadas e repassadas aos pacientes nas atividades desenvolvidas pelos acadêmicos durante a graduação, em relação à motivação, é muito satisfatória ou satisfatória, enquanto que $20,10 \%$ a consideraram regular. Esse dado está de acordo com pesquisa realizada na UNIFOR, na qual $91,5 \%$ dos entrevistados consideraram o aprendizado nas disciplinas de Saúde Bucal coletiva como muito satisfatório ou satisfatório, bem como, a grande maioria considerou adequado o enfoque dado à disciplina. ${ }^{11}$

A relação dentista-paciente/população provavelmente é um reflexo da relação alunoprofessor, a qual se estabelece de forma vertical. ${ }^{12}$ Boa parte dos cirurgiões-dentistas, atuantes no serviço público, não realiza atividades voltadas para a educação em saúde. ${ }^{13}$ Para eles, os temas conhecidos com mais frequência são a prevenção e a educação, o que indica uma visão preventivista nas ações que se propõem a desenvolver. ${ }^{14}$

Apesar de 96,34\% dos estudantes considerarem muito importante a orientação do paciente quanto às práticas de higiene bucal, incentivando-os a manter hábitos saudáveis, percebemos que mais de $20 \%$ não o fazem. Esse dado é inferior a dados observados nos serviços públicos onde estão inseridos os egressos, cerca $47,8 \%$ não desenvolvem práticas em saúde coletiva, entre elas a orientação do paciente; foi percebido que o fato de recordar os assuntos abordados na universidade não necessariamente influenciam no desenvolvimento dessas atividades. ${ }^{2}$ Provavelmente, esse fato esteja relacionado ao desligamento do meio acadêmico, uma vez que a influência exercida pelos docentes comprometidos em incentivar esse modelo de formação do acadêmico e atendimento ao paciente diminui; dessa forma, cabe ao então profissional optar ou não por seguir os modelos estabelecidos durante sua graduação.

As práticas educativas em saúde foram identificadas como um instrumento de mudança de comportamento, que leva a população a adquirir hábitos de vida considerados saudáveis para a prevenção das doenças bucais. ${ }^{15}$

Essa dificuldade em exercer práticas participativas e dialógicas, com os pacientes, em educação em saúde, pode ser reflexo da própria formação acadêmica dos profissionais de saúde, que geralmente é pautada em metodologias de ensino-aprendizagem centradas no professor, fazendo que se reproduza nos serviços o mesmo modelo pedagógico que eles foram formados. ${ }^{16,17}$

Sendo assim, a maneira como os futuros profissionais adquirem os conhecimentos necessários às suas práticas clínicas e educativas revela a influência do meio acadêmico como também de aspectos sociais, políticos, culturais e econômicos. A principal fonte de informações da população acerca do tema saúde bucal e a maneira adequada de prevenção dos problemas bucais, em diferentes países, é o cirurgião-dentista, representando $57 \%, 90 \%, 82,3 \%, 35,9 \%$, $78 \%$ e $39,3 \%$, respectivamente, em pesquisas realizadas por Glasrud, Frazier (1988), ${ }^{18}$ Lang et al. (1989), ${ }^{19}$ Petersen et al. (1990), ${ }^{20}$ Oliveira (1996), ${ }^{21}$ al-Tamimi, Petersen $(1998)^{22}$ e SganCohen et al. (1999). ${ }^{23}$

A principal fonte de orientação sobre higiene 
bucal, entre alunos do primeiro ano, foi de origem familiar (70\%). Já entre os alunos do quinto ano, a maioria teve como principal fonte de orientação as aulas na universidade, $52,9 \% .^{24}$

À medida que os períodos avançam, as principais fontes de conhecimentos para os alunos são a graduação e cursos, palestras, simpósios ou congressos que já participaram. A busca por mais informações ou por aprimoramento do conhecimento de alunos mais próximos da conclusão do curso caracteriza meios secundários de aquisição destes, principalmente na literatura disponível e atualizações em eventos.

Para que se possa intervir com segurança, é necessário que se conheça a etiologia e o tratamento adequado das patologias bucais, em especial das mais frequentes. Sabe-se que outros fatores também estão relacionados ao desenvolvimento de certas patologias, mas algumas estão diretamente relacionadas às condições de higiene do paciente podendo, ou não, ter associação com outros fatores.

A maioria dos participantes soube diferenciar as patologias que estavam diretamente relacionadas com a higiene bucal. Um número expressivo assinalou Cárie, Doença Periodontal e Língua Saburrosa (167, 154 e 133 participantes, respectivamente). A média de períodos foi entre 5,56 e 6,12. Foram encontrados resultados significativos somente para as patologias Doença Periodontal e Língua Saburrosa.

Todavia, é preciso informar, motivar e educar os acadêmicos para torná-los conscientes, não apenas da importância do conhecimento técnico-científico, mas também sobre a necessidade da mudança de hábitos e do seu papel educador na sociedade. Tal orientação torna-se necessária, visto que os conhecimentos adquiridos e as atitudes formadas durante a graduação são incorporados na prática profissional e podem influenciar a qualidade dos cuidados dispensados aos pacientes. ${ }^{25}$

Após o ingresso do estudante no curso de Odontologia, $77,5 \%$ relataram mudança nos hábitos pessoais de higiene bucal, enquanto $22,5 \%$ afirmaram não ter tido mudança. Possivelmente, os alunos que estão nos períodos iniciais e que ainda não tiveram contato com disciplinas que abordam essa temática ainda pratiquem os hábitos com os quais conviveram até o momento; sendo assim, essa porcentagem tão significativa deve estar associada a esse fator. Temos que observar também que os alunos, assim como os pacientes, são influenciados por elementos sociais, culturais, econômicos, entre outros e que a mudança de comportamento é alcançada gradativamente.

Os instrumentos utilizados com maior frequência pelos alunos foram antisséptico, dentifrício, escova macia e fio dental. Neste estudo, $58,6 \%$ dos participantes afirmaram escovar os dentes três vezes ao dia e 50,3\% utilizam fio dental apenas uma vez ao dia. As análises demonstram que há relacionamento significativo entre a frequência de escovação e o uso de antisséptico, sugerindo que aumenta a chance de utilização do último quando se tem uma frequência maior de escovação.

Esse dado corrobora com pesquisa realizada por Oliveira (2007), ${ }^{24}$ na qual foi observado que todos os alunos analisados relataram escovar os dentes pelo menos três vezes ao dia. Entretanto, alunos do primeiro ano tiveram a maior frequência de escovação diária (55\% dos entrevistados escovavam acima de quatro vezes por dia). Aproximadamente a metade dos alunos do quinto ano afirmou ter frequência de escovação menor, três vezes ao dia. Em relação ao uso de fio ou fita dental a maioria dos alunos utilizava pelo menos uma vez ao dia. ${ }^{26}$

Os resultados obtidos merecem reflexão, tendo em vista que a percepção do acadêmico 
a respeito do perfil de formação dos cirurgiõesdentistas, bem como, a aplicabilidade e o enfoque que são dados aos itens relacionados à Saúde Coletiva durante toda a sua graduação devem ser conhecidas e avaliadas. A despeito da motivação e do impacto gerados nas atitudes do acadêmico, tanto em relação às práticas de orientação e motivação dos seus pacientes quanto no que diz respeito às práticas e mudanças de hábitos pessoais de higiene bucal, ainda é preciso a realização de mais estudos, já que são poucas as avaliações do perfil do estudante de Odontologia. O incentivo à realização de novos estudos está ligado ao fato de que é preciso conhecer a realidade de cada instituição, para que assim se observe a coerência do modelo de formação e a percepção e satisfação dos alunos com relação a essas práticas.

\section{Considerações finaIS}

Foi observado que a totalidade dos acadêmicos concorda com o fato da importância e relevância dos assuntos relacionados à Saúde/Higiene bucal, como também, que mais da metade avalia como satisfatória a abordagem sobre esse tema realizada na instituição que estão inseridos. Apesar disso, ainda é preciso aumentar o incentivo às práticas de educação em saúde efetuadas por eles e conhecer mais detalhadamente o perfil de formação, o impacto ocasionado em suas condutas clínicas e hábitos pessoais durante a graduação e a base de seus conhecimentos no tocante às práticas de Saúde Coletiva.

\section{REFERÊNCIAS}

1. Sales TI, et al. Percepções de estudantes de graduação em Odontologia sobre o Sistema de Saúde Brasileiro. Rev da ABENO, 2016; 16 (2): 69-76. https://revabeno.emnuvens.com.br/ revabeno/article/download/265/221
2. Bandan DEC, Marcelo VC, Rocha DG. Percepção e utilização dos conteúdos de saúde coletiva por cirurgiões-dentistas egressos da Universidade Federal de Goiás. Ciência \& Saúde Coletiva, 2010; 15 (Supl. 1): 1.811-1.818. www.scielo.br/scielo.php?pid=S1413$-81232010000700093 \&$ script=sci...tlng.

3. Vignavajah S. Oral health knowledge and behaviours and barriers to dental attendance of school children and adolescents in the Caribbean island of Antigua. Int Dent J., 1997; 47: 167-72. https://www.ncbi.nlm.nih.gov/pubmed/9448803

4. Santos PA dos, Rodrigues J. de A., Garcia PPNS. Avaliação do conhecimento e comportamento de saúde bucal de professores de ensino fundamental da cidade de Araraquara. J Bras Odontopediatr Odontol Bebê, 2003; 6 (33): 389-97. https://www.dtscience.com/.../Avaliação-do-Conhecimento-e-Comportamento-de-Saúde

5. Unfer B., Saliba O. Avaliação do conhecimento popular e práticas cotidianas em saúde bucal. Revista de Saúde Pública, São Paulo, 2000; 2 (34): 190-195.

www.scielo.br/scielo.php?script=sci_arttext\&pid =S0034-89102000000200014

6. Guerra MJC, et al. Impact of oral health conditions on the quality of life of workers, 2014; Ciência \& Saúde Coletiva, 19 (12): 4.777-4.786.

www.scielo.br/pdf/csc/v19n12/pt_1413-8123csc-19-12-04777

7. Rocha PML, Dinelli W. Odontologia em saúde coletiva: percepção do acadêmico. Revista Científica Vozes dos Vales, 2015; (8): 1-12.

site.ufvjm.edu.br/revistamultidisciplinar/files/2015/11/Joao

8. Santos LM, et al. Self-perception on oral health and its relationship with use of services and prevalence of tooth ache. Revista Ciência Plural, 2016; 2 (2): 14-27.

https://periodicos.ufrn.br/rcp/article/download/9470/7842 
9. Milanezi LA, Garcia VG, Milanezi FM, Teodoro LH. Atitudes e modelos preventivos de higienização bucal desenvolvidos pelos cirurgiões-dentistas. Reg Araçatuba Assoc Paul Cir Dent, 2003; 24: 43-46.

http://apcdaracatuba.com.br/revista/2402/ pag43-46.

10. Koyama, DFV. Avaliação da formação do cirurgião-dentista em uma instituição pública, 70 s. Dissertação (Mestrado em Odontologia) - Universidade Estadual de Londrina, Londrina, 2012.

$<$ http://www.bibliotecadigital.uel.br/ document/?code=vtls000185600>.

11. Noro LRA, Torquato SM. Percepção sobre o aprendizado de saúde coletiva e o SUS entre alunos Concludentes de curso de odontologia Trab. Educ. Saúde, Rio de Janeiro, 2011; 8 (3): 439-447.

www.scielo.br/scielo.php?script=sci_arttext\&pid $=$ S1981-77462010000300006

12. Reis, SMAS, Cicillini, GA. Práticas docentes no ensino odontológico: aproximações e distanciamentos das diretrizes curriculares nacionais. Revista Ibero-Americana de Estudos em Educação, Araraquara, v. 6, n. 2, p. 1-15, 2011. http://www.seer.fclar.unesp.br/iberoamericana/ article/view/4878>.

13. Araújo YP, Dimenstein M. Estrutura e organização do trabalho do cirurgião-dentista no PSF de municípios do Rio Grande do Norte. Cien Saude Colet, 2006; 11 (1): 219-227.

www.scielo.br/scielo.php?script=sci_arttext\&pid $=$ S1413-81232006000100031

14. Guterman N. O cirurgião dentista como educador em saúde: explorações em torno de uma prática [dissertação]. Natal (RN): UFRN; 2002.

15. Mialhe FL, Silva CMC. A educação em saúde e suas representações entre alunos de um curso de odontologia. Ciência \& Saúde Coletiva, 2011; 16 (Supl. 1): 1.555-1.561.

www.scielo.br/scielo.php?script=sci_arttext\&pid $=$ S1413-81232011000700091
16. Akhter R., Hassan NM, Martin EF, Muhit M., Haque MR, Smithers-Sheedy H. et al. Risk factors for dental caries among children with cerebral palsy in a low-resource setting, 2016; Dev Med Child Neurol.

https://www.ncbi.nlm.nih.gov/pubmed/27935024

17. Silva MES, Villaça EL, Magalhães CS, Ferreira EF. Impacto da perda dentária na qualidade de vida. Cien Saude Colet, 2010; 15 (3): 841-850. www.scielo.br/scielo.php?script=sci_arttext\&pid $=$ S1413-81232010000300027

18. Glasrud PH, Frazier PJ. Future elementary schoolteachers' knowledge and opinions about oral health and community programs. J Public Health Dent., 1988; 48 (2): 74-80.

https://www.ncbi.nlm.nih.gov/pubmed/3164402

19. Sanders AE, Slade GD, Lim S., Reisine ST. Impact of oral disease on quality of life in the US and Australian populations. Community Dent Health 2009; 37 (2): 171-181.

https://www.ncbi.nlm.nih.gov/pubmed/19175659

20. Cohen-Carneiro F., Souza-Santos R., Rebelo MAB. Quality of life related to oral health: contribution from social factors. Cien Saude Colet. 2011; 16 (Supl.1): 1.007-1.015.

https://www.ncbi.nlm.nih.gov/pubmed/21503449

21. Silva SRC, Fernandes RAC. Autopercepção das condições de saúde bucal por idosos. Rev Saude Publica, 2001; 35 (4): 349-355.

www.scielo.br/scielo.php? pid=S0034$89102001000400003 \&$ script=sci.

22. Al-Tamimi S., Petersen PE. Oral health of schoolchildren, mothers and schoolteachers in Saudi Arabia. Int Dent J., 1998; 48 (3): 180-6. https://www.ncbi.nlm.nih.gov/pubmed/9779097

23. Sales Peres SHC, Theodoro DS, Ribeiro DA, Avila ED, Greghi GA, Silva RPR. Odontologia do trabalho: doenças e lesões na prática profissional. Rev Fac Odontol Araçatuba, 2006; 27 (1): 54-58.

pesquisa.bvsalud.org/cvsp/resource/pt/biblio-856774 ?lang $=p t$ 
24. Moimaz SAS, Saliba NA, Saliba O. \& Almeida JCF. Educação para saúde bucal e prevenção. Avaliação entre cirurgiões-dentistas de serviço público e particular. RGO., 1994; 42 (2): 71-74. www.scielo.br/scielo.php?script $=$ sci_ nlinks\&ref=000131\&pid=S1413...Ing

25. Freire MCM, Dias HRP, Souza CS. Hábitos e atitudes dos acadêmicos de odontologia da Universidade Federal de Goiás em relação ao açúcar. Rev Odontol Univ São Paulo, 1997; 11: 221-7.

www.scielo.br/scielo.php?script=sci_arttext\&pid $=$ S0103-06631997000300011

26. Cortelli SC, Chaves MGAM, Faria IS, Landucci LF, Oliveira LD, Scherma AP, Jorge AOC. Avaliação da condição bucal e do risco de cárie de alunos ingressantes em curso de Odontologia. PGR-Pós-Grad Rev Fac Odontol São José dos Campos, 2002; 5 (1): 35-42.

https://www.researchgate.net/publication/237751421_Avaliacao_da_condicao_ bucal_e_do_risco_de_carie_de_alunos_ingressantes_em_curso_de_Odontologia_1

\section{AUTOR CORRESPONDENTE: \\ Rafaella Bastos Leite}

Programa de Pós-graduação em Patologia Oral - Universidade Federal do Rio Grande do Norte, Natal-RN, Brasil.

Av. Salgado Filho, 1787, Lagoa Nova - Natal/RN. CEP: 59056-000.

Telefone: +55 $8432154138 /$ +55 83998092884 .

E-mail: rrafaella_bastos@hotmail.com

Rafaella Bastos Leite ${ }^{1}$

Joana Emília Araújo dos Santos Furtado ${ }^{2}$

Kátia Simone Alves dos Santos ${ }^{1}$

Raquel Cristina Barboza Gomes ${ }^{1}$

Carlos Augusto Galvão Barboza ${ }^{3}$

Carlos Frederico de Moraes Sarmento ${ }^{1}$

${ }^{1}$ Professor (a) - Departamento de Odontologia da Universidade Estadual da Paraíba, Campina Grande-PB, Brasil.

${ }^{2}$ Graduanda - Departamento de Odontologia da Universidade Estadual da Paraíba, Campina Grande-PB, Brasil.

Submetido em: 26-5-2018

Aceito em: 28-8-2018 